\title{
Effects of personality on the association between paroxetine plasma concentration and response
}

This article was published in the following Dove Press journal:

Neuropsychiatric Disease and Treatment

\author{
Tetsu Tomita' \\ Norio Yasui-Furukori' \\ Taku Nakagami² \\ Shoko Tsuchimine ${ }^{3}$ \\ Masamichi Ishioka ${ }^{4}$ \\ Ayako Kaneda' \\ Kazuhiko Nakamura' \\ 'Department of Neuropsychiatry, \\ Graduate School of Medicine, \\ Hirosaki University, Hirosaki, \\ Japan; ${ }^{2}$ Department of Psychiatry, \\ Nakagami Mental Clinic, Odate, Japan; \\ ${ }^{3}$ Department of Mental Disorder \\ Research, National Institute of \\ Neuroscience, National Center of \\ Neurology and Psychiatry, Tokyo, \\ Japan; ${ }^{4}$ Department of Psychiatry, \\ Minato Hospital, Hachinohe, Japan
}

Correspondence: Tetsu Tomita Department of Neuropsychiatry, Graduate School of Medicine, Hirosaki University, 5 Zaifu-cho, Hirosaki, Aomori 036-8562, Japan

Tel +8I I 72395066

Fax +8I I72395067

Email ttomita1984@yahoo.co.jp
Background: We studied the differences between groups that were divided according to personality characteristics with respect to the relationship between drug concentration and symptom improvement.

Methods: A total of 120 patients with major depressive disorder were treated with paroxetine for 6 weeks, and 89 patients completed the protocol. The Montgomery-Åsberg Depression Rating Scale (MADRS) was used to evaluate the patients. Patients' paroxetine plasma concentrations at week 6 were measured. Their personalities were evaluated by the Temperament and Character Inventory (TCI) at the first visit. We divided the patients into two groups according to the median of each TCI dimension. We compared the responder rate between "high" and "low" groups in each TCI dimension and analyzed Pearson's correlation coefficients of paroxetine plasma concentration and MADRS-improvement rate.

Results: A total of 62 patients completed the TCI. Low-novelty-seeking, high-harm-avoidance, low-reward-dependence, and low-self-directedness groups exhibited significant negative correlations between paroxetine plasma concentration and MADRS improvement. Among the groups with combined personality traits, the high-harm-avoidance and low-self-directedness groups showed a markedly significant negative correlation.

Conclusion: Patients with depression exhibiting specific personality traits, especially those with high harm-avoidance and low self-directedness scores, exhibited a significant negative association between paroxetine plasma concentration and MADRS-improvement rate. Therefore, a lower dose might be suitable for patients with specific personality traits.

Keywords: depression, paroxetine, concentration, personality, Temperament and Character Inventory, TCI

\section{Introduction}

In the field of clinical psychopharmacology, a lot of studies on the treatment of depression have tried to clarify associations between drug levels and therapeutic response. Some studies have reported probable therapeutic reference ranges of drug concentration and the probability of a statistical association between drug levels and therapeutic response, but almost all these studies focused on typical tricyclic antidepressants. ${ }^{1-4}$ Although these studies may reveal the advantage of considering drug levels, patients with depression do not benefit from this fact during depression treatment in clinical situations, especially those treated with antidepressants, except for tricyclic antidepressants. Some studies on patients with depression treated with paroxetine or selective serotonin-reuptake inhibitors have shown a probable therapeutic window and an upper concentration threshold. ${ }^{5-7}$

Previously, we investigated the relationship between treatment response and paroxetine plasma concentration in two groups that were divided according to their serotonin 
transporter-linked polymorphic region (5HTTLPR) genotype. We reported a difference between positive/negative associations among patients with the SS genotype and L carriers: the SS group exhibited a negative association between concentration and symptom improvement, whereas the L carriers exhibited a positive association. ${ }^{8}$ It is possible that some patient factors may influence the efficacy of antidepressant dosage or that dosage may be increased or decreased for patients who do not show a sufficiently strong response to drug treatment, according to genetic information related to depression or neurotransmitters that are thought to be related to depression.

The personality of depressive patients might be related to both the efficacy and the genetic factors of the patients. Cloninger et al suggested that some divisions of the Temperament and Character Inventory (TCI) are related to neurotransmitters. ${ }^{9,10}$ Many studies have reported associations between the characteristics of the TCI and the therapeutic response or clinical course among patients with depression. Many studies have reported a depressive mood associated with higher harm avoidance (HA) and lower self-directedness (SD) scores. ${ }^{11-19}$ High HA scores are most strongly associated with depression or depressive states. ${ }^{11,14-19}$ In addition, many studies have shown associations between some dimensions of the TCI and genetic or biological characteristics. Peirson et al indicated that $5 \mathrm{HT}_{2}{ }^{-}$ receptor sensitivity is positively associated with HA scores and negatively associated with SD scores. ${ }^{20}$ Other reports showed that an association between novelty-seeking (NS) scores and dopamine $\mathrm{D}_{2}$-receptor-binding potential and between NS scores and heterozygosity for the short allele of the dopamine $\mathrm{D}_{4}$-receptor gene in a female sample..$^{21,22}$

As such, information on the drug concentrations and genes of patients with depression might be beneficial, but it is difficult to measure antidepressant concentrations and investigate genetic information in a clinical setting. Therefore, in the present study, we used information on the personalities of patients with depression, which may be related to genetic features, instead of actual genetic information, and studied the differences between groups divided according to characteristics of their personalities in terms of the relationship between drug concentration and symptom improvement.

\section{Methods}

\section{Patients}

Patients with major depressive disorder according to the fourth edition of the Diagnostic and Statistical Manual of Mental Disorders were included for this study. They were aged 18-70 years. Those with scores $<20$ points on the Montgomery-
Åsberg Depression Rating Scale (MADRS) ${ }^{23}$ were excluded from the study. The MADRS consists of ten items, each of which is scored on a scale of 0-6. Patients had been free of medications, including any psychotropic agents, for at least 1 month before the initiation of the study. Those with clinically significant abnormal laboratory or electrocardiography findings, a history of a mental disorder other than major depressive disorder (eg, bipolar affective disorder, schizophrenia, epilepsy, alcoholism, or drug abuse), or any clinically significant organic or neurological disease were excluded from the study. This study was approved by the ethics committee of Hirosaki University Hospital, and written, informed consent prior to participation were obtained from all patients. All procedures were performed in accordance with the Declaration of Helsinki. A total of 120 patients were initially enrolled in the study, and 89 completed it (34 men and 55 women): 31 dropped out because of severe side effects (17), undetectable plasma drug concentrations (four), not completing required hospital visits for unknown reasons (seven), and withdrawn consent for personal reasons (three).

\section{Protocol}

During the first visit (in the morning), blood samples $(10 \mathrm{~mL})$ were taken after a 30-minute rest. Clinical status before the study using the structured interview guide for the MADRS to assess depressive symptoms and the Udvalg für Kliniske Undersogelser (UKU) side-effect-rating scale ${ }^{24,25}$ were assessed by two well-trained psychiatrists.

Paroxetine $20 \mathrm{mg} /$ day was initiated at $8 \mathrm{pm}$ for week 1 , and the dose was escalated to $40 \mathrm{mg}$ /day during weeks 2-6. In the case of mild side effects (UKU score 1), the dose was maintained. The paroxetine dose was reduced in the case of moderate side effects (UKU score 2) and its administration discontinued if severe side effects (UKU score 3) occurred. No other drugs were prescribed, except for diazepam (2-5 mg/day, $\mathrm{n}=19)$ for anxiety, brotizolam $(0.25 \mathrm{mg} /$ day, $\mathrm{n}=20$, or $4 \mathrm{mg} / \mathrm{day}, \mathrm{n}=17$ ) for insomnia, and sennoside $(12-48 \mathrm{mg} /$ day, $\mathrm{n}=12)$ as a laxative to treat constipation. Blood samples were taken during treatment weeks 1, 2, and 6. Clinical symptoms were evaluated using the MADRS and the UKU scale during treatment weeks 1, 2, 4, and 6.

The 240-item Japanese version of the TCI, the reliability and validity of which have been established, was performed at the beginning of the study. ${ }^{26}$ The TCI consists of seven dimensions: four related to temperament (NS, HA, reward dependence [RD], and persistence), and three related to character (SD, cooperativeness), and self-transcendence. In total, 62 of the 89 patients completed the TCI. 


\section{Assays for paroxetine}

Plasma levels of paroxetine at week 6 were quantified using HPLC, described in other studies. ${ }^{7,8,27,28}$ The lowest limits of detection and quantification were 0.5 and $1.0 \mathrm{ng} / \mathrm{mL}$, respectively, and intra-assay and interassay coefficients of variation were $<10 \%$ at all calibration-curve concentrations $(1-150 \mathrm{ng} / \mathrm{mL})$ of paroxetine.

\section{Data analysis and statistics}

We defined improvement rate as MADRS score at week 6 divided by MADRS score at baseline. We defined responders as patients with MADRS improvements $>50 \%$ from baseline at week 6 and responder rate as the rate of responders of patients completed the protocol. A $t$-test and $\chi^{2}$ test were performed to compare demographic and clinical data between responders and nonresponders. We divided the 62 patients into two groups according to the median of each TCI dimension (eg, high NS, low NS). We compared responder rates between high and low groups in each TCI dimension and analyzed Pearson's correlation coefficients for all groups.

According to the characteristics that were associated with response based on the analysis, we divided patients into two groups: patients with and without combined characteristics associated with a response (eg, high NS, low RD). Similarly, we compared responder rates between high and low groups for each TCI dimension and analyzed Pearson's correlation coefficients for all groups. $P<0.05$ was considered to indicate statistical significance. All analyses were performed using SPSS 22 for Windows (IBM, Armonk, NY, USA).

\section{Results}

Among the 62 patients, 43 showed a response. Table 1 shows the demographic and clinical data of these patients. There was no significant difference in age or sex between responders and nonresponders. The plasma concentration of paroxetine in responders was lower than in nonresponders. Among the TCI dimensions, only cooperativeness was significantly different, for which responders showed significantly higher scores.

Median NS, HA, RD, persistence, SD, cooperativeness, and self-transcendence scores were 18, 28, 14, 3, 20, 27, and 10 , respectively, and we divided patients into two groups (high and low) for each TCI dimension, according to the medians. Table 2 shows rates of responders and correlations between paroxetine concentration and MADRS-improvement rate among patients divided according to their scores in each TCI dimension. There was no significant difference in responder rates between the high and low groups in any TC dimension. However, low-NS, high-HA, low-RD, and low-SD groups showed significant negative correlations between paroxetine plasma concentration and MADRS improvement.

Table 3 shows responder rates and correlations between paroxetine concentration and MADRS-improvement rate among patients with and without the aforementioned

Table I Demographic and clinical data of patients

\begin{tabular}{l|l|l|l|l}
\hline & Entire cohort $\mathbf{( n = 6 2 )}$ & Responders $(\mathbf{n}=\mathbf{4 3})$ & Nonresponders $(\mathbf{n}=\mathbf{1 9})$ & $\mathbf{P}$-value \\
\hline Age, years & $46.4 \pm 13.0$ & $47.6 \pm 12.6$ & $43.8 \pm 13.9$ & 0.299 \\
Sex (M:F) & $22: 40$ & $13: 30$ & $9: 10$ & 0.194 \\
MADRS, week & $39.8 \pm 9.2$ & $39.8 \pm 9.0$ & $39.8 \pm 9.7$ & 0.998 \\
0 & $28.5 \pm 10.9$ & $25.8 \pm 10.2$ & $34.5 \pm 10.1$ & $0.003^{* *}$ \\
I & $20.3 \pm 11.7$ & $17.9 \pm 10.9$ & $25.8 \pm 11.8$ & $0.013^{*}$ \\
2 & $15.4 \pm 12.2$ & $10.1 \pm 8.9$ & $27.5 \pm 9.7$ & $0.000^{* *}$ \\
4 & $13.2 \pm 12.8$ & $6.1 \pm 5.5$ & $29.4 \pm 9.6$ & $0.000^{* *}$ \\
6 & $64.7 \pm 45.0$ & $53.8 \pm 42.6$ & $89.5 \pm 41.3$ & $0.003^{* *}$ \\
Concentration & & & & \\
TCI & $17.3 \pm 4.5$ & $17.8 \pm 4.3$ & $16.1 \pm 4.9$ & 0.168 \\
NS & $27.7 \pm 4.0$ & $27.3 \pm 3.8$ & $28.6 \pm 4.2$ & 0.233 \\
HA & $13.8 \pm 3.3$ & $14.1 \pm 3.4$ & $13.2 \pm 3.0$ & 0.321 \\
RD & $3.7 \pm 1.7$ & $3.5 \pm 1.6$ & $4.0 \pm 2.0$ & 0.286 \\
P & $20.6 \pm 6.2$ & $21.3 \pm 6.4$ & $19.1 \pm 5.8$ & 0.213 \\
SD & $26.9 \pm 4.4$ & $27.8 \pm 3.9$ & $24.6 \pm 4.7$ & $0.007^{* *}$ \\
C & $10.7 \pm 5.3$ & $11.3 \pm 5.3$ & $9.5 \pm 5.4$ & 0.235 \\
ST & & & \\
\hline
\end{tabular}

Notes: $* P<0.05 ; * * P<0.01$.

Abbreviations: C, cooperativeness; HA, harm avoidance; MADRS, Montgomery-Åsberg Depression Rating Scale; NS, novelty-seeking; P, persistence; RD, reward dependence; SD, self-directedness; ST, self-transcendence; TCl, Temperament and Character Inventory. 
Table 2 Responders and correlations between paroxetine concentration and MADRS-improvement rate by patient scores in each $\mathrm{TCl}$ dimension

\begin{tabular}{|c|c|c|c|c|c|c|}
\hline & MADRS, week 0 & $P$-value & $\begin{array}{l}\text { n (responders, } \\
\text { nonresponders) }\end{array}$ & $P$-value & $\begin{array}{l}\text { Correlation } \\
\text { coefficients }\end{array}$ & $P$-value \\
\hline Entire & $39.8 \pm 9.2$ & - & $62(43,19)$ & - & -0.304 & $0.016 *$ \\
\hline \multicolumn{7}{|l|}{ NS } \\
\hline Low & $41.7 \pm 8.9$ & 0.137 & $29(19,10)$ & 0.539 & -0.370 & $0.048 *$ \\
\hline High & $38.2 \pm 9.2$ & & $33(24,9)$ & & -0.263 & 0.139 \\
\hline \multicolumn{7}{|l|}{$\mathrm{HA}$} \\
\hline Low & $38.2 \pm 10.0$ & 0.232 & $26(20,6)$ & 0.272 & -0.072 & 0.729 \\
\hline High & $41.0 \pm 8.5$ & & $36(23,13)$ & & -0.514 & $0.001 * *$ \\
\hline \multicolumn{7}{|l|}{ RD } \\
\hline Low & $41.6 \pm 6.9$ & 0.142 & $29(18,11)$ & 0.243 & -0.420 & $0.023 *$ \\
\hline High & $38.3 \pm 10.6$ & & $33(25,8)$ & & -0.178 & 0.321 \\
\hline \multicolumn{7}{|l|}{$P$} \\
\hline Low & $38.6 \pm 9.4$ & 0.238 & $33(24,9)$ & 0.539 & -0.286 & 0.107 \\
\hline High & $41.3 \pm 8.8$ & & $29(19,10)$ & & -0.338 & 0.073 \\
\hline \multicolumn{7}{|l|}{ SD } \\
\hline Low & $43.4 \pm 7.4$ & $0.004^{* *}$ & $28(18,10)$ & 0.432 & -0.496 & $0.007^{* *}$ \\
\hline High & $36.9 \pm 9.5$ & & $34(25,9)$ & & -0.253 & 0.148 \\
\hline \multicolumn{7}{|l|}{ C } \\
\hline Low & $41.9 \pm 10.1$ & 0.116 & $28(16,12)$ & 0.058 & -0.223 & 0.255 \\
\hline High & $38.2 \pm 8.1$ & & $34(27,7)$ & & -0.324 & 0.062 \\
\hline \multicolumn{7}{|l|}{ ST } \\
\hline Low & $37.0 \pm 10.4$ & $0.033^{*}$ & $27(16,11)$ & 0.130 & -0.293 & 0.138 \\
\hline High & $42.0 \pm 7.6$ & & $35(27,8)$ & & -0.242 & 0.162 \\
\hline
\end{tabular}

Notes: $* p<0.05 ; * * p<0.01$

Abbreviations: C, cooperativeness; HA, harm avoidance; MADRS, Montgomery-Åsberg Depression Rating Scale; NS, novelty-seeking; P, persistence; RD, reward dependence; SD, self-directedness; ST, self-transcendence; TCI, Temperament and Character Inventory.

Table 3 Responder rates and correlations between paroxetine concentration and MADRS-improvement rate in patients with/without combined characteristics

\begin{tabular}{|c|c|c|c|c|c|c|}
\hline & MADRS, week 0 & $P$-value & $\begin{array}{l}\text { n (responders, } \\
\text { nonresponders) }\end{array}$ & $P$-value & $\begin{array}{l}\text { Correlation } \\
\text { coefficients }\end{array}$ & $P$-value \\
\hline \multicolumn{7}{|l|}{ Low NS-high HA } \\
\hline Applicable & $42.5 \pm 7.3$ & 0.095 & $22(14,8)$ & 0.469 & -0.477 & $0.025 *$ \\
\hline Not applicable & $38.4 \pm 9.8$ & & $40(29,11)$ & & -0.233 & 0.147 \\
\hline \multicolumn{7}{|l|}{ Low NS-low RD } \\
\hline Applicable & $41.9 \pm 8.0$ & 0.274 & I5 $(I I, 4)$ & 0.701 & -0.473 & 0.075 \\
\hline Not applicable & $39.2 \pm 9.5$ & & $47(32,15)$ & & -0.297 & $0.042 *$ \\
\hline \multicolumn{7}{|l|}{ Low NS-low SD } \\
\hline Applicable & $45.2 \pm 6.8$ & $0.008^{* *}$ & $15(10,5)$ & 0.795 & -0.547 & $0.035^{*}$ \\
\hline Not applicable & $38.1 \pm 9.2$ & & $47(33,14)$ & & -0.247 & 0.094 \\
\hline \multicolumn{7}{|l|}{ High HA-low RD } \\
\hline Applicable & $41.2 \pm 6.8$ & 0.451 & $18(11,7)$ & 0.368 & -0.472 & $0.048 *$ \\
\hline Not applicable & $39.3 \pm 10.0$ & & $44(32,12)$ & & -0.220 & 0.152 \\
\hline \multicolumn{7}{|l|}{ High HA-low SD } \\
\hline Applicable & $43.4 \pm 7.8$ & $0.026 *$ & $21(13,8)$ & 0.362 & -0.633 & $0.002^{* *}$ \\
\hline Not applicable & $38.0 \pm 9.3$ & & $4 I(30, I I)$ & & -0.196 & 0.219 \\
\hline \multicolumn{7}{|l|}{ Low RD-low SD } \\
\hline Applicable & $42.4 \pm 6.5$ & 0.201 & $16(9,7)$ & 0.187 & -0.541 & $0.031 *$ \\
\hline Not applicable & $39.0 \pm 9.8$ & & $46(34,12)$ & & -0.251 & 0.093 \\
\hline
\end{tabular}

Notes: Applicable, subjects with each combined characteristic; not applicable, subjects without each combined characteristic; $* P<0.05 ; * * P<0.01$.

Abbreviations: C, cooperativeness; HA, harm avoidance; MADRS, Montgomery-Åsberg Depression Rating Scale; NS, novelty-seeking; P, persistence; RD, reward dependence; SD, self-directedness; ST, self-transcendence. 
combined characteristics, including the combination of any two characteristics among low NS, high HA, low RD, and low SD. There was no significant difference in responder rate between patients with and without the combined characteristics. Patients with characteristics of low NS-high HA, low NS-low SD, high HA-low RD, high HA-low SD, and low RD-low SD exhibited significant negative correlations between paroxetine plasma concentration and MADRS improvement. The high HA-low SD group exhibited a relatively high correlation coefficient ( $r=-0.633, P=0.002$ ). Only the low NS-low RD and remaining patient groups showed a significant negative correlation between paroxetine plasma concentration and MADRS improvement.

\section{Discussion}

In the present study, we divided patients with depression treated by paroxetine into groups according to their personality traits evaluated by the TCI and studied the relationship between paroxetine plasma concentration and MADRS improvement at the end of the protocol. We thought that combined characteristics meant stricter conditions and being able to define more clearly patients with positive or negative correlations between paroxetine levels and improvement. Overall, patients with higher or lower scores in some dimensions of the TCI, especially those with high HA and low SD scores, showed a significantly negative association between paroxetine plasma concentration and improvement rate, while the others showed no significant associations. Therefore, it may be feasible to decrease or increase the dose of paroxetine or other antidepressants according to the patient's personality traits. Some studies have reported that personality trait or temperament might influence the outcome of treatment for patients with depression. ${ }^{29-32}$ Specific drugs we reported to show lower efficacy in patients with specific personality traits, and Reiner and Spangler showed that specific personality traits might associated with adherence. ${ }^{32}$ Previous studies showed the influence of personality and temperament on clinical course. This study is the first to show the probable efficacy of tailoring a treatment plan by considering the personality of a patient with depression.

In the present study, responders showed significantly lower paroxetine concentrations than nonresponders, but the results were not consistent with previous studies reporting that higher paroxetine concentration was related to poor response. ${ }^{5,7}$ Meyer et al reported that serotonin-transporter occupancy was elevated and reached a plateau with elevation of paroxetine concentration, and this mechanism might be related to the relationship between low paroxetine concentration and response. ${ }^{33}$ It is not unclear why nonresponders showed higher concentrations of paroxetine, but some mild side effects (eg, nausea, sedation) might make the nonresponders associate bad condition with depression.

It is unclear why patients with high HA or low SD scores exhibited a negative association between paroxetine plasma concentration and MADRS improvement rate. In a previous study investigating changes of TCI traits pre- and posttreatment for patients with depression using paroxetine, only SD scores of nonresponders increased significantly, and others did not show significant change. ${ }^{34}$ Although we did not find a reason for the results in this previous study, the mechanisms might have been similar to the negative associations in the present study. As mentioned previously, it has been reported that $5 \mathrm{HT}_{2}$-receptor sensitivity is positively associated with HA scores and negatively associated with SD scores. ${ }^{20}$ Another study showed that postsynaptic $5 \mathrm{HT}_{2 \mathrm{~A}}$ receptors are downregulated by antidepressants. This mechanism might be associated with the expression of antidepressive effects. ${ }^{35}$ The influence of higher sensitivity of $5 \mathrm{HT}_{2}$ receptors in patients with higher HA or lower SD scores may have induced a relationship between paroxetine plasma concentration and MADRS-improvement rate.

Previous research has studied the association between temperament or personality and genetic characteristics with respect to treatment response or characteristics in the treatment course. ${ }^{36-38}$ Studies have reported that patients with depression of the SS genotype or S allele of 5HTTLPR exhibited a specific personality trait or that a genetic characteristic and score were associated with their clinical characteristics. ${ }^{35,38}$ An association between the genetic characteristics of 5HTTLPR and the plasma concentration of paroxetine in treatment response has been suggested. ${ }^{8}$ Additional information on the plasma concentration of drugs may be incorporated into a predictive model, including personality traits and genotype, for predicting treatment response in patients with depression. ${ }^{37}$ Iniesta et al reported a predictive model using multiple genetic information for treatment response using escitalopram and nortriptyline. ${ }^{39}$ In future studies, predictive model using genetic and personality information might be established.

The high-HA, low-SD, and high HA-low-SD groups showed strong and significant negative relationships between plasma concentration and MADRS-improvement rate. Higher HA scores and lower SD scores have been reported to be associated with depression or depressed mood, so patients with these characteristics might be "typical". ${ }^{11-19}$ 
As mentioned previously, these dimensions are thought to be related to serotonin and noradrenaline. $5 \mathrm{HT}_{2}$-receptor sensitivity is associated with HA and SD scores, and HA score is suggested to indicate central serotonergic turnover. ${ }^{10,20}$ The low-SD and high-HA-low-SD groups showed significantly lower baseline MADRS scores than the others, but there was no significant difference in the responder rate; therefore, these characteristics may not be associated with treatment resistance. High HA and low SD scores may reflect characteristics of neurotransmitter-mechanism status, serotonergic turnover, and serotonin sensitivity, ${ }^{10,20}$ and a lower concentration of antidepressants may be suitable for this group, as a higher concentration of paroxetine would not likely improve their neurotransmitter mechanisms.

The low-NS and low-RD groups showed significantly negative correlations between paroxetine plasma concentration and MADRS improvement, but patients with combined characteristics of low NS-low RD did not show a significant difference. Moreover, patients without combined traits showed significant results with an opposite trend, as the other characteristics contributed more strongly to a significant negative correlation between paroxetine plasma concentration and MADRS improvement. NS scores have been reported to be associated with dopamine, ${ }^{10,21,22}$ and hence this score may be more important in other psychiatric disorders or features of depression.

The present study has some limitations. First, it is unclear whether differences in the significance of the relationships between paroxetine plasma concentration and improvement in symptoms in personality traits are dependent on genetics. We discussed genetic reasons for these differences, but a placebo effect of lower plasma concentrations may be present for specific personality traits. Second, the dropout rate was relatively high, and there may have been a selection bias among the subjects. We were able to study only those patients who had completed the TCI and protocol, and the results might apply only to those patients. In addition, the sample size was relatively low, and the findings of the present study do not apply to general subjects. Third, we studied only paroxetine. If the results of the present study reflect the genetic characteristics associated with serotonin, then studies on other classes of antidepressants might provide different results based on genetic characteristics associated with dopamine or noradrenaline, which would result in character traits that are different from those in the present study.

\section{Conclusion}

Patients with depression exhibiting specific personality traits, especially those with high HA and low SD scores, showed a significant negative association between paroxetine plasma concentration and MADRS-improvement rate. We might use a lower dose for patients with specific personality traits.

\section{Abbreviations}

TCAs, tricyclic antidepressants; 5HTTLPR, serotonintransporter-linked polymorphic region; TCI, Temperament and Character Inventory; NS, novelty-seeking; HA, harm avoidance; RD, reward dependence; $\mathrm{P}$, persistence; SD, selfdirectedness; C, cooperativeness; ST, self-transcendence; MADRS, Montgomery-Åsberg Depression Rating Scale; UKU, Udvalg für Kliniske Undersogelser.

\section{Ethics and consent to participate}

Approval was obtained from the ethics committee of the Hirosaki University School of Medicine prior to the study. The participants provided written informed consent after receiving a full description of the study.

\section{Acknowledgments}

The authors would like to thank all their coworkers in this study for their skillful assistance in collecting and managing the data. This study was funded by a Grant-in-Aid for Scientific Research (Kakenhi) from the Japan Society for the Promotion of Research (JSPS, 20333734 and 15H04754), Mitsubishi Pharma Research Foundation, Asteras Schizophrenia Research Foundation, and a grant from the Hirosaki Research Institute for Neurosciences. The funders had no role in study design, data collection/analysis, decision to publish, or preparation of the manuscript.

\section{Author contributions}

NY-F and KN conceived and designed the experiments, NY-F, TN, ST, AK, and MI performed the experiments, and TT analyzed the data and wrote the paper. All authors contributed to data analysis, drafting and revising the article, gave final approval of the version to be published, and agree to be accountable for all aspects of the work.

\section{Disclosure}

NY-F has received grant/research support or honoraria from and been a speaker for Astra, Dainippon, Eli Lilly, GSK, Janssen-Pharma, Meiji, Mochida, MSD, Otsuka, Pfizer, Takata, and Yoshitomi. The authors report no other conflicts of interest in this work.

\section{References}

1. Amsterdam J, Brunswick D, Mendels J. The clinical application of tricyclic antidepressant pharmacokinetics and plasma levels. Am J Psychiatry. 1980;137(6):653-662. 
2. Tricyclic antidepressants - blood level measurements and clinical outcome: an APA Task Force report. Task Force on the Use of Laboratory Tests in Psychiatry. Am J Psychiatry. 1985;142(2):155-162.

3. Perry PJ, Pfohl BM, Holstad SG. The relationship between antidepressant response and tricyclic antidepressant plasma concentrations. A retrospective analysis of the literature using logistic regression analysis. Clin Pharmacokinet. 1987;13(6):381-392.

4. Perry PJ, Zeilmann C, Arndt S. Tricyclic antidepressant concentrations in plasma: an estimate of their sensitivity and specificity as a predictor of response. J Clin Psychopharmacol. 1994;14(4):230-240.

5. Yasui-Furukori N, Nakagami T, Kaneda A, et al. Inverse correlation between clinical response to paroxetine and plasma drug concentration in patients with major depressive disorders. Hum Psychopharmacol. 2011;26(8):602-608.

6. Hiemke C, Baumann P, Bergemann N, et al. AGNP consensus guidelines for therapeutic drug monitoring in psychiatry: update 2011. Pharmacopsychiatry. 2011;44(6):195-235.

7. Tomita T, Yasui-Furukori N, Nakagami T, et al. Therapeutic reference range for plasma concentrations of paroxetine in patients with major depressive disorders. Ther Drug Monit. 2014;36(4):480-485.

8. Tomita T, Yasui-Furukori N, Nakagami T, et al. The influence of 5-HTTLPR genotype on the association between the plasma concentration and therapeutic effect of paroxetine in patients with major depressive disorder. PLoS One. 2014;9(5):e98099.

9. Cloninger CR. A systematic method for clinical description and classification of personality variants. A proposal. Arch Gen Psychiatry. 1987;44(6):573-588.

10. Cloninger CR, Svrakic DM, Przybeck TR. A psychobiological model of temperament and character. Arch Gen Psychiatry. 1993;50(12): 975-990.

11. Brown SL, Svrakic DM, Przybeck TR, Cloninger CR. The relationship of personality to mood and anxiety states: a dimensional approach. J Psychiatr Res. 1992;26(3):197-211.

12. Bayon C, Hill K, Svrakic DM, Przybeck TR, Cloninger CR. Dimensiona assessment of personality in an out-patient sample: relations of the systems of Millon and Cloninger. J Psychiatr Res. 1996;30(5):341-352.

13. Cloninger CR, Bayon C, Svrakic DM. Measurement of temperament and character in mood disorders: a model of fundamental states as personality types. J Affect Disord. 1998;51(1):21-32.

14. Hansenne M, Reggers J, Pinto E, Kjiri K, Ajamier A, Ansseau M. Temperament and character inventory (TCI) and depression. JPsychiatr Res. 1999;33(1):31-36.

15. Naito M, Kijima N, Kitamura T. Temperament and Character Inventory (TCI) as predictors of depression among Japanese college students J Clin Psychol. 2000;56(12):1579-1585.

16. Richter J, Eisemann M, Richter G. Temperament and character during the course of unipolar depression among inpatients. Eur Arch Psychiatry Clin Neurosci. 2000;250(1):40-47.

17. Farmer A, Mahmood A, Redman K, Harris T, Sadler S, McGuffin P. A sib-pair study of the Temperament and Character Inventory scales in major depression. Arch Gen Psychiatry. 2003;60(5):490-496.

18. Spittlehouse JK, Pearson JF, Luty SE, et al. Measures of temperament and character are differentially impacted on by depression severity. $J$ Affect Disord. 2010;126(1-2):140-146.

19. Kaneda A, Yasui-Furukori N, Nakagami T, Sato Y, Kaneko S. The influence of personality factors on paroxetine response time in patients with major depression. J Affect Disord. 2011;135(1-3):321-325.

20. Peirson AR, Heuchert JW, Thomala L, Berk M, Plein H, Cloninger CR. Relationship between serotonin and the temperament and character inventory. Psychiatry Res. 1999;89(1):29-37.

21. Suhara T, Yasuno F, Sudo Y, et al. Dopamine D2 receptors in the insular cortex and the personality trait of novelty seeking. Neuroimage. 2001;13(5):891-895.
22. Lee HJ, Lee HS, Kim YK. D2 and D4 dopamine receptor gene polymorphisms and personality traits in a young Korean population. Am J Med Genet B Neuropsychiatr Genet. 2003;121b(1):44-49.

23. Montgomery SA, Asberg M. A new depression scale designed to be sensitive to change. Br J Psychiatry. 1979;134:382-389.

24. Takahashi N, Tomita $\mathrm{K}$, Higuchi $\mathrm{T}$, Inada $\mathrm{T}$. The inter-rater reliability of the Japanese version of the Montgomery-Asberg depression rating scale (MADRS) using a structured interview guide for MADRS (SIGMA). Hum Psychopharmacol. 2004;19(3):187-192.

25. Lingjaerde O, Ahlfors UG, Bech P, Dencker SJ, Elgen K. The UKU side effect rating scale. A new comprehensive rating scale for psychotropic drugs and a cross-sectional study of side effects in neuroleptic-treated patients. Acta Psychiatr Scand Suppl. 1987;334:1-100.

26. Kijima N, Saito R, Takeuchi M. Clininger's seven-factor model of temperament and character and Japanese version of Temperament and Character Inventory (TCI). Archives of Psychiatric Diagnostics and Clinical Evaluation. 1996;7(3):379-399.

27. Yasui-Furukori N, Saito M, Inoue Y, et al. Terbinafine increases the plasma concentration of paroxetine after a single oral administration of paroxetine in healthy subjects. Eur J Clin Pharmacol. 2007;63(1): 51-56.

28. Yasui-Furukori N, Saito M, Niioka T, Inoue Y, Sato Y, Kaneko S. Effect of itraconazole on pharmacokinetics of paroxetine: the role of gut transporters. Ther Drug Monit. 2007;29(1):45-48.

29. Joffe RT, Regan JJ. Personality and response to tricyclic antidepressants in depressed patients. J Nerv Ment Dis. 1989;177(12):745-749.

30. Joyce PR, Mulder RT, Cloninger CR. Temperament predicts clomipramine and desipramine response in major depression. J Affect Disord. 1994;30(1):35-46.

31. Peselow ED, Fieve RR, Difiglia C. Personality traits and response to desipramine. J Affect Disord. 1992;24(4):209-216.

32. Reiner I, Spangler G. Dopamine D4 receptor exon III polymorphism, adverse life events and personality traits in a nonclinical German adult sample. Neuropsychobiology. 2011;63(1):52-58.

33. Meyer JH, Wilson AA, Sagrati S, et al. Serotonin transporter occupancy of five selective serotonin reuptake inhibitors at different doses: an [11C] DASB positron emission tomography study. Am J Psychiatry. 2004; 161(5):826-835

34. Tomita T, Kaneda A, Nakagami T, Kaneko S, Yasui-Furukori N. Changes in the Temperament and Character Inventory dimensions after paroxetine treatment in patients with major depressive disorder. Hum Psychopharmacol. 2015;30(5):334-340.

35. Gray JA, Roth BL. Paradoxical trafficking and regulation of 5-HT(2A) receptors by agonists and antagonists. Brain Res Bull. 2001;56(5): $441-451$.

36. Andre K, Kampman O, Illi A, et al. SERT and NET polymorphisms, temperament and antidepressant response. Nord J Psychiatry. 2015; 69(7):531-538.

37. Nishioka G, Yashima H, Kiuchi Y, et al. Prediction and structural equation model of sertraline treatment response in Japanese patients with major depressive disorder. Hum Psychopharmacol. 2013;28(6): 576-585.

38. Minelli A, Bonvicini C, Scassellati C, Sartori R, Gennarelli M. The influence of psychiatric screening in healthy populations selection: a new study and meta-analysis of functional 5-HTTLPR and rs25531 polymorphisms and anxiety-related personality traits. BMC Psychiatry. 2011;11:50.

39. Iniesta R, Hodgson K, Stahl D, et al. Antidepressant drug-specific prediction of depression treatment outcomes from genetic and clinical variables. Sci Rep. 2018;8(1):5530. 


\section{Publish your work in this journal}

Neuropsychiatric Disease and Treatment is an international, peerreviewed journal of clinical therapeutics and pharmacology focusing on concise rapid reporting of clinical or pre-clinical studies on a range of neuropsychiatric and neurological disorders. This journal is indexed on PubMed Central, the 'PsycINFO' database and CAS,

and is the official journal of The International Neuropsychiatric Association (INA). The manuscript management system is completely online and includes a very quick and fair peer-review system, which is all easy to use. Visit http://www.dovepress.com/testimonials.php to read real quotes from published authors.

Submit your manuscript here: http://www.dovepress.com/neuropsychiatric-disease-and-treatment-journal 